\title{
Soil microbial biomass and bacterial diversity in Southern European regions vulnerable to desertification
}

\section{Catania Valentina}

University of Palermo

\section{Rafael Silveira Bueno}

University of Palermo

\section{Alduina Rosa \\ University of Palermo}

Eleonora Grilli

University of Campania "Luigi Vanvitelli"

Tommaso La Mantia

University of Palermo

\section{Simona Castaldi}

University of Campania "Luigi Vanvitelli"

Paola Quatrini ( $\sim$ paola.quatrini@unipa.it )

University of Palermo

\section{Research Article}

Keywords:

Posted Date: February 3rd, 2022

DOI: https://doi.org/10.21203/rs.3.rs-1306993/v1

License: (9) (i) This work is licensed under a Creative Commons Attribution 4.0 International License. Read Full License 


\section{Abstract}

Soil functionality is strongly dependent on the soil microbiota, which in turn is affected by soil quality and climate. Among global change factors, desertification is the most threatening ecosystem change affecting southern Europe, but the effects on the soil microbiota are largely unknown. In this study, we investigated soil microbial biomass and bacterial diversity in regions of southern European countries (Spain, Portugal and Italy), most under desertification risk, and related to key soil chemical-physical indicators and land use. Soil microbial biomass was positively related, to soil organic carbon (SOC) but bacterial diversity was negatively correlated with it. $\mathrm{pH}$ was the most influencing factor affecting soil alpha-diversity, while SOC, $\mathrm{pH}$ and cation exchange capacity drive the biogeographic patterns of bacterial communities. $16 \mathrm{~S}$ metagenomics allowed the identification of a core microbiota of the analyzed semiarid soils, including Proteobacteria, Actinobacteria, Acidobacteria and Firmicutes. We identified, at regional scale, a few rare multi-extremophilic endemic genera, which could reveal functional strategies to be exploited for arid land restoration. This work creates the baseline for the necessary monitoring of soils facing climate change and for the evaluation of the efficacy of adaptation measures in semi-arid European soils.

\section{Introduction}

Mediterranean soils are experiencing increasingly negative effects of climate change due to drought and extreme weather phenomena ${ }^{1}$. Such climate change effects are exacerbated by intensive agriculture and overgrazing, that are inducing soil erosion, compaction, salinization and loss of soil functional properties, a critical issue particularly on dry and semi-dry conditions covering large regions of the Mediterranean 23 . Dry conditions are generally estimated by the Aridity Index (Al: the ratio of annual precipitation to annual potential evapotranspiration) and Al values between 0.2 and 0.5 indicate semi-arid regions ${ }^{4}{ }^{5}$. In such regions, soil degradation can rapidly degenerate to desertification, with profound changes on soil physico-chemical properties such as organic matter reduction and biodiversity loss ${ }^{6}{ }^{3}$. SOC reduction is of particular concern because, beyond being the major source of nutrients, it exerts numerous positive effects on soil physicochemical properties as well as on soil's regulatory ecosystem services, including the reduction of $\mathrm{CO}_{2}$ emissions ${ }^{7}$. Such changes are expected to cause also a dramatic shift in the microbial diversity and biomass although the magnitude of this influence is still far from being understood ${ }^{89}$.

SOC depletion is usually associated with a lower soil biological activity and diversity but no studies report a specific threshold level of SOC associated to a critical dysfunctionality of the soil microbial community

3 . Recently, a SOC concentration of $20 \mathrm{~g} \mathrm{~kg}^{-1}$ was indicated as a threshold value below which any additional decline of SOC might result in a steep variation of many key soil parameters in semi-arid regions ${ }^{10}$, indicating that this concentration is critical for soil ecosystem services. 
SOC turnover is dependent on soil microbial community dynamics ${ }^{11} 1112$ and the ratio between the fraction of $C$ retained in microbial biomass and the $C$ fraction that is lost by microbial heterotrophic respiration (referred to as carbon use efficiency CUE) is expected to be reduced under dry conditions and high temperatures ${ }^{13}$. Thus, global warming in semi-arid regions is expected to increase the respiration rates of soil microbes because the processes they mediate are temperature sensitive, thus contributing to increase carbon loss to the atmosphere ${ }^{14}$. On the other hand, increased temperature can also increase rates of microbial turnover in soil, increasing the stability of soil organic matter ${ }^{15}$.

There is growing awareness of the fundamental role played by the soil microbiota, with its highly diverse and unique metabolisms and functions, in providing ecosystem services that support soil quality and plant productivity 1617182 . For this reason monitoring soil biodiversity, has been encouraged as a method of assessing soil quality and health and to inform management and policy to contrast climate change, especially in semi-arid regions ${ }^{19}$. Actually, the patterns of microbial biomass and soil bacterial diversity in relationship to the main soil biotic and abiotic features, land use, biogeography and global change factors are still far to be known ${ }^{20}$. Below-ground microbial diversity seems not to respond consistently to the abiotic environment ${ }^{21}$, indicating that much remains to be learned about drivers of below-ground diversity, especially in arid soils. Moreover, many of the drivers co-vary and feedback on each other ${ }^{20}$ thus it is still unclear which are potential drivers of microbial diversity and which factors are instead regulated by below-ground diversity.

In Europe, a recent study has reinforced the contrasting relationship between soil physicochemical properties, land use and bacterial diversity, also supporting spatial structuration of bacterial communities 22. The paper provides also specific bacterial indicators of land use and soil conditions but only four sampling sites were located in the Mediterranean, and only two of them in areas under desertification risk 22.

The present study specifically targets on soils threatened by desertification in Mediterranean climatic areas of southern Europe ${ }^{23}$, with the aim to: i) investigate the relationships between soil chemicalphysical parameters and land use type with microbial biomass and bacterial diversity; ii) identify the core microbiota characterizing the European semi-arid soils; iii) create the baseline data to subsidize the monitoring of bacterial communities in soils facing climate change and desertification. For this purpose, soil microbial biomass and bacterial diversity were estimated on a large set of soils from vulnerable regions of Italy (Sicily), Spain (Extremadura) and Portugal (Alentejo), most of which characterized by an Aridity index below 0.5 , where an assessment of chemical-physical parameters was previously carried out ${ }^{10}$. A molecular fingerprinting method based on ribosomal 16S-23S genes intergenic spacer length polymorphisms (Automated Ribosomal Intergenic Spacer Analysis, ARISA) was used to determine bacterial diversity, while a more in depth analysis of the bacterial community structure and composition was done on a subset of soils by high throughput metagenomics 16S rRNA gene sequencing. Microbial biomass and diversity were related to the main soil chemical-physical parameters ( $\mathrm{pH}, \mathrm{SOC}, \mathrm{CEC}, \mathrm{TN})$, and land cover. 


\section{Results And Discussion}

\section{Soil microbial biomass}

The total soil microbial biomass (SMB) of 86 soil samples from 30 study sites (Table S1, Fig. S1) across

southern Europe Mediterranean regions was estimated using total soil dsDNA as a proxy (Table S2) 2425 . The amount of extracted metagenomic dsDNA from soils of the study sites ranged from 7.09 to $208.6 \mu \mathrm{g}$ $\mathrm{g}^{-1}$ soil dry weight (Table S2) and varied significantly across sites (Fig.S2a. Kruskall-Wallis $\chi^{2}=11.42, \mathrm{p}=$ 0.003), with soils of Extremadura (SP) presenting significantly higher SMB than soils from Sicily (IT) and Alentejo (PT) (Dunn test $p<0.05$; Fig. S2). SMB was positively related to SOC and also to TN (Table 1) coherently with the positive correlation between SOC and TN (Plassart et al., 2019; Grilli et al, 2021). SMB was higher in non-arid sites (Fig. S3, soils with Al $>0.65$, Wilcoxon $X^{2}=11.61, p<0.001$ ), as a result of higher plant productivity, increased access to substrates due to soil moisture, and higher microbial growth rates, in accordance with the results found in other studies ${ }^{8}{ }^{26}$. SMB also varied across cover with coniferous stand soils displaying the highest SMB, and croplands the lowest (Dunn test $p<0.05$, (Fig. 1, Table S2). Microbes in croplands are generally affected by cultivation practices that disrupt soil aggregates, where they live, and by inorganic fertilization, that increases nutrient availability, promoting microbial community shifts towards more copiotrophic taxa, with higher respiration rates; ${ }^{27}$. Since most SOC formation is mediated by microbial activity ${ }^{28}$, a reduction in microbial biomass and, eventually, in its substrate use efficiency, in tilled soils, may diminish the overall potential for SOC formation ${ }^{29}$. For these reasons sustainable soil management, based on reduced tillage, organic fertilization and cover crops, could help to maintain crop yield while avoiding further SOC depletion in semi-arid soils ${ }^{30}$.

Interestingly high SMB was detected under the coniferous forest of the driest investigated area in Lampedusa (IT1, aridity index: 0.29), where high SOC levels were also detected ${ }^{10}$ in agreement with other studies on SOC under pine tree woods ${ }^{31}$. The levels of microbial biomass under coniferous stands is controversial because other studies report that soils under pine plantations, in semi-arid soils, present markedly smaller and less active microbial communities 3233 , generally explained by the lower availability and degradability of organic substrates, such as lignin ${ }^{24}$. However the involvement of bacteria in wood litter decomposition including cellulose and aromatic compounds derived from lignin degradation is more important than was previously assumed ${ }^{35}$. As bacteria, more than fungi, can modulate at community level their carbon use efficiency, the specific composition of the soil microbiota could explain SOC accumulation in dry soils. Drought could act on bacteria by reducing the microbial respiration rate ${ }^{36}$ and increasing the stability of soil organic matter ${ }^{15}$. Moreover bacteria could persist in soils for long periods in a dormancy condition as seed banks and be revived when environmental conditions change 377 . All this could contribute to higher resilience to drought of coniferous forest ecosystems in respect to other soil uses ${ }^{38} 39$. We found the lowest SMB levels in soils with $\mathrm{SOC}<20 \mathrm{~g} \mathrm{~kg}$ ${ }^{-1}$ (Wilcoxon $Z=-4.73, p<0.001$ ), the threshold value below which any additional decline of SOC 
concentrations might result in a steep variation of most soil parameters ${ }^{10}$, indicating that this concentration is also critical for key microbe-driven soil ecosystem services ${ }^{9}$.

\section{Relationship between bacterial diversity and edaphic variables}

Molecular fingerprinting of the soil bacterial communities, was obtained by the automated ribosomal intergenic spacer analysis (ARISA) on a subset of 69 soil DNA samples from the 30 study sites. Average OTU richness ranged from 9.0 (S.E. 0.5) to 46.5 (0.26) and Shannon index ranged from 2.34 (0.26) to 3.55 (0.2) (Table S2). Soil bacterial richness and Shannon index significantly differed across the sites (Fig. S2, ANOVA F = 33.08, $p<0.001$, Kruskall-Wallis $\chi^{2}=20,68, p<0.001$ ) with soils of Sicily presenting higher richness than all the other sites ( $p<0.001$ after Tukey HSD). Richness and Shannon index also varied across land cover $\left(F=4.79, p=0.002 ; X^{2}=21,76, p<0.001\right)$, although only for croplands and shrublands values were significantly different (after Tukey HSD; Fig. 1). Bacterial richness was higher in coniferous soils while Shannon index was higher in croplands; both indexes showed the lowest values in shrubland soils.

Among the analyzed soil variables, $\mathrm{pH}$ was the most influencing factor affecting soil bacterial alphadiversity with significantly positive relationships $(p<0.001)$, conversely it had no significant effect on SMB, which was positively related with SOC (Table 1).

Bacterial diversity tended to be higher in the low-SOC soils ( $\mathrm{SOC}<20 \mathrm{~g} \mathrm{~kg}^{-1}$ ), where Shannon diversity reached the highest values $(Z=2.28, p=0.02)$, while bacterial richness was not significantly influenced by low SOC levels $(t=-1.72, p=0.08)$. Coherently, semi-arid sites $(A l<0.65)$ presented higher bacterial diversity (richness $t=-5,04, p<0.0001$; Shannon index $X^{2}=11.78, p<0.001$ ) than non-arid sites $(A l>0.65)$ (Fig. S4). Several studies have reported such a negative relation between soil bacterial diversity and total $\mathrm{C}$, leading to the conclusions that, a decline in SOC, as a result of soil degradation and aridity, leads to an increased bacterial diversity 14041 especially in arid soils ${ }^{9}$. In the semi-arid soils, however, a large part of the biomass, contributing to the extracted dsDNA, could be dormant, thus metagenomics could overestimate the real expressed soil functional microbial diversity. In this respect metaproteomics could be a better predictive indicator of ecosystem functionality than DNA diversity ${ }^{37}$.

Soil TN was inversely related to both bacterial OTU richness and Shannon index (Table 1). TN is not always considered an explanatory variable for microbial diversity ${ }^{22}$ although our data suggest that it can have a stronger negative relationship with bacterial diversity than SOC.

The Canonical Analysis of Principal coordinates (CAP) indicated that beta-diversity is related to the main soil parameters ${ }^{10}$ (Fig. 2), mainly $\mathrm{pH}$, SOC and CEC, as already reported in other studies ${ }^{141}$.

The similarities among the bacterial communities across all sites reflected their biogeography confirming that soil bacterial communities were spatially structured 352222 . Interestingly, the coniferous forest soils (SP2-1 and IT1-2) displayed the highest distance from their respective biogeographic cluster, indicating the strong influence on the soil bacterial assemblage of this soil cover, in respect to the others. 
Table 1. Summary of the regression testing the effects of $\mathrm{SOC}, \mathrm{pH}, \mathrm{N}$ and $\mathrm{CEC}$ on soil microbial biomass $(\mathrm{SMB})$, richness (estimated as observed bacterial OTUs, $\mathrm{n}=86$ ), and diversity (Shannon index) calculated on ARISA profiles and measured as soil extracted dsDNA ( $n=69) . R^{2}=$ adjusted fit of the model. In bold characters

\begin{tabular}{llllll} 
& & Estimate & $\mathrm{SE}$ & $\mathrm{t}$ & $\mathrm{p}$ \\
\hline $\mathrm{SMB}$ & Intercept & -19.156 & 28.005 & -0.68 & 0.496 \\
\hline $\mathrm{R}^{2}=44$ & $\mathrm{SOC}$ & 0.572 & 0.177 & 3.23 & $\mathbf{0 . 0 0 2}$ \\
\hline & $\mathrm{pH}$ & 7.013 & 4.089 & 1.72 & 0.091 \\
\hline & $\mathrm{TN}$ & 10.012 & 3.974 & 2.52 & $\mathbf{0 . 0 1 4}$ \\
\hline & $\mathrm{CEC}$ & -0.889 & 0.798 & -1.11 & 0.270 \\
\hline Richness & Intercept & -3.368 & 6.723 & -0.50 & 0.618 \\
\hline $\mathrm{R}^{2}=0.49$ & SOC & 0.099 & 0.042 & 2.33 & 0.023 \\
\hline & $\mathrm{pH}$ & 4.676 & 0.982 & 4.76 & $<0.001$ \\
\hline & $\mathrm{TN}$ & -2.249 & 0.954 & -2.36 & 0.021 \\
\hline & $\mathrm{CEC}$ & 0.334 & 0.192 & 1.74 & 0.086 \\
\hline Shannon & Intercept & 2.498 & 0.240 & 10.393 & $<0.001$ \\
\hline $\mathrm{R}^{2}=0.21$ & $\mathrm{SOC}$ & 0.002 & 0.001 & 1.648 & 0.105 \\
\hline & $\mathrm{pH}$ & 0.091 & 0.035 & 2.612 & $\mathbf{0 . 0 1 1}$ \\
\hline & TN & -0.068 & 0.034 & -2.022 & 0.048 \\
\hline & $\mathrm{CEC}$ & 0.005 & 0.007 & 0.712 & 0.480
\end{tabular}

\section{The soil microbiota of European Mediterranean soils}

Illumina sequencing of the 16S rRNA gene was carried out on a subset of 22 soil DNA samples. The subset was selected to include different soil covers in the three regions, and two replicates $(a$ and $b)$ for each site. Only one sample (IT1-2 replicate b) gave no results, due to low quality DNA that hampered further processing. The subset of 21 soil DNA samples was sequenced and resulted (after denoising and chimeras check) in a minimum of 13,482 to a maximum of 73,358 reads, with an average of 44,922 reads for each sample (Table S3). Richness, assessed on bacterial observed OTUs clustered at $97 \%$, ranged from 107 to 581 OTU $_{97}$ (Table S3), both extremes were observed in two croplands in central Sicily (Italy). OTU ${ }_{97}$ richness, was positively correlated to the alpha diversity based on ARISA profiles (Pearson tests $r=0.36, p=0.12)$ considering all data, and the correlation improved $(r=0.56, p=0.013)$ excluding 
the outlier IT4-1a. This significant correlation shows that the two data sets of bacterial diversity were congruent ${ }^{42}$.

All soils contained the main typical soil bacterial phyla ${ }^{40}$ but with different abundances in respect to European soils of other climatic zones ${ }^{22}$ and also in respect to decertified soils of other continents ${ }^{41}$.

The most abundant bacterial phyla were Proteobacteria (average relative abundance $26.6 \%$ ), Actinobacteria (24.2\%), Acidobacteria (16.6), Firmicutes (11.3), Bacteroidetes (5.5), Chloroflexi (5.4) and Verrucomicrobia (5.0) (Fig. 3). The dominance level of these phyla is more similar to previous characterizations of world's dry forests and drylands ${ }^{40}$ than non-Mediterranean European soils ${ }^{22}$. In Southern European soils Proteobacteria were less abundant, and not always the dominant phylum, while Actinobacteria and Acidobacteria were much more abundant than in higher European latitudes ${ }^{22}$. Archaea were below $2 \%$, mainly represented by Crenarchaeota. Beyond Proteobacteria, the phyla Acidobacteria, Verrucomicrobia and Chloroflexi were more abundant in the soils of Extremadura (SP) and Alentejo (PT) while Actinobacteria were more abundant in Sicilian soils (IT) (Fig. S3). The overall relative abundance of the 7 main phyla did not vary across soils (Kruskal-Wallis $X^{2}=0.76, p=0.68$ ) or land use $\left(X^{2}=1.09, p=0.89\right)$. We did not find any significant difference in the relative abundance of each phylum among the five different land cover (ANOVA and Kruskal-Wallis $p>0.05$ ). Considering each phylum separately, however, the abundance of 5 out of the 7 dominant phyla was significantly related to cation exchange capacity (CEC), with positive and negative relationships (Table 2). Soil CEC is considered strongly correlated with the organic carbon content ${ }^{22}$ as well as to the physical (structural stability), chemical (nutrient availability), and biological characteristics of soils ${ }^{43}$. Four phyla were related to $\mathrm{pH}$, two positively, Firmicutes and Verrucomicrobia and two negatively, Acidobacteria and Chloroflexi. Interestingly, the phylum Firmicutes was significantly related to all edaphic variables (SOC, pH, TN, and $\mathrm{CEC}$ ), conversely Proteobacteria was not significantly related to any of the analyzed variables. In particular the relative abundance of Firmicutes was negatively related to SOC, in agreement with the role of Firmicutes as responsible for enhanced mineralization of dissolved organic carbon and increased soil respiration 4445 .

Table 2. Summary of the OLS and GLM models testing the effects of cation exchange capacity (CEC), pH, total nitrogen (TN) and soil organic carbon (SOC) on the relative abundances of the 7 most abundant phyla. In bold characters the significant values $(p<0.05)$. 


\begin{tabular}{|c|c|c|c|c|c|}
\hline Phylum & & Estimate & SE & $t / z$ & $\mathbf{p}$ \\
\hline Acidobacteria & Intercept & 26.78 & 4.25 & 6.30 & $<001$ \\
\hline & CEC & -0.28 & 0.11 & -2.49 & 0.024 \\
\hline & $\mathrm{pH}$ & -1.60 & 0.68 & -2.35 & 0.032 \\
\hline & $\mathrm{TN}$ & 0.42 & 0.42 & 0.98 & 0.340 \\
\hline & SOC & 0.04 & 0.02 & 1.76 & 0.098 \\
\hline Actinobacteria & Intercept & -5.66 & 9.41 & -0.60 & 0.556 \\
\hline & CEC & 0.67 & 0.25 & 2.68 & 0.016 \\
\hline & $\mathrm{pH}$ & 2.97 & 1.51 & 1.97 & 0.066 \\
\hline & $\mathrm{TN}$ & -1.23 & 0.94 & -1.31 & 0.210 \\
\hline & SOC & 0.05 & 0.04 & 1.10 & 0.287 \\
\hline Bacteroidetes & Intercept & 1.57 & 0.99 & 1.58 & 0.114 \\
\hline & CEC & 0.05 & 0.03 & 1.97 & 0.049 \\
\hline & $\mathrm{pH}$ & -0.04 & 0.16 & -0.23 & 0.821 \\
\hline & $\mathrm{TN}$ & -0.10 & 0.10 & -1.01 & 0.313 \\
\hline & SOC & 0.00 & 0.00 & -0.46 & 0.648 \\
\hline Chloroflexi & Intercept & 6.46 & 0.92 & 6.98 & $<001$ \\
\hline & CEC & 0.07 & 0.03 & 2.69 & 0.007 \\
\hline & $\mathrm{pH}$ & -0.70 & 0.16 & -4.39 & $<001$ \\
\hline & $\mathrm{TN}$ & -0.11 & 0.09 & -1.30 & 0.194 \\
\hline & soc & -0.01 & 0.00 & -2.21 & 0.027 \\
\hline Firmicutes & Intercept & 0.55 & 1.06 & 0.52 & 0.602 \\
\hline & CEC & -0.11 & 0.03 & -3.95 & $<001$ \\
\hline & $\mathrm{pH}$ & 0.39 & 0.16 & 2.37 & 0.018 \\
\hline & $\mathrm{TN}$ & 0.39 & 0.12 & 3.19 & 0.001 \\
\hline & SOC & -0.02 & 0.01 & -2.37 & 0.018 \\
\hline Proteobacteria & Intercept & 17.09 & 7.16 & 2.39 & 0.030 \\
\hline & CEC & 0.16 & 0.19 & 0.84 & 0.413 \\
\hline & $\mathrm{pH}$ & 0.41 & 1.15 & 0.36 & 0.727 \\
\hline
\end{tabular}




\begin{tabular}{clllll} 
& TN & -0.02 & 0.72 & -0.03 & 0.979 \\
\hline & SOC & 0.05 & 0.03 & 1.57 & 0.136 \\
\hline Verrucomicrobia & Intercept & 17.37 & 3.40 & 5.11 & $<001$ \\
\hline & CEC & 0.02 & 0.09 & 0.21 & 0.836 \\
\hline & pH & -1.87 & 0.54 & -3.43 & 0.003 \\
\hline & TN & 0.20 & 0.34 & 0.60 & 0.557 \\
\hline & SOC & -0.03 & 0.02 & -1.76 & 0.098
\end{tabular}

Conversely, Acidobacteria and Chloroflexi, more abundant in acidic soils of Spain and Portugal, under broad leaved agroforest, have low carbon turnover and, consequently, low carbon dioxide emission and higher carbon sequestration capacity ${ }^{46}$. In soils of Sicily the most abundant phylum was Actinobacteria ${ }^{7}$. The definition of this phylum as copiotroph or oligotroph is controversial. Some studies associate it with copiotrophy, others suggest that it is better suited to oligotrophic conditions probably because it includes members that are able to use both labile and recalcitrant substrates ${ }^{47}$. In areas with SOC below $20 \mathrm{~g} \mathrm{~kg}^{-1}$ Actinobacteria $(t=2.70, p=0.014)$, Bacteroidetes $(Z=-2.10, p=0.035)$ and Proteobacteria $(t=2.91, p=0.012)$ were significantly less abundant suggesting a copiotrophic lifestyle of these three phyla, including Actinobacteria.

The correlation of edaphic variables with soil bacterial diversity and lifestyle, however, depends on the taxonomic level considered ${ }^{47}$. In fact, we observed that some critical parameters, as CEC, can have no correlation with phyla as a result of positive and negative relations with taxa at the order level within the same phylum (Table S4). In general, the correlation between the chemico-physical parameters and the five most abundant orders within each phylum, confirmed the prevalent role of $\mathrm{pH}$ in modulating the distribution of bacterial taxa in soils, with positive and negative correlations (Table S4). Within Actinobacteria two orders (Acidimicrobiales and Solirubrobacterales) showed positive and significant relation to SOC, but, in contrast, the sole genus Streptomyces highly abundant in low SOC soils showed negative correlation to SOC and also to CEC. Within Proteobacteria, Rhodospirillales and Rhizobiales were instead positively related to SOC suggesting that they may contribute to $\mathrm{C}$ sequestration in soils of semiarid regions. Only $38 \%$ of the sequences (on average) was identified at the genus level (Fig. 4).

Most of the genera with abundancies above $1 \%$ were correlated to soil pH with the exclusion of those within the phylum Firmicutes (Table 3). Conversely, in the Firmicutes, 4 out of the 6 most abundant genera (Bacillus, Ammoniphilus, Solibacillus and Sporosarcina) were negatively correlated to SOC and 3 also to CEC, with Ammoniphylus and Bacillus showing highly significant negative correlations (Table 3). The negative correlation with SOC of genera in the Firmicutes, was also confirmed at the order level (Table S4). Bacillus and Ammoniphilus, are known copiotrophs meaning that they rapidly consume the 
substrate ${ }^{48}$ thus giving low contribution to SOC accrual. The genus Bacillus in particular, was dominant in low-SOC pastures, broad-leaved forest soils of Portugal and in a cropland of central Sicily, where Opuntia ficus indica is organically cultivated with no-tillage and mulching using Opuntia cladodes (M. Russo personal communication). The abundancy of copiotrophs in this cropland could be the result of abundant fresh organic matter (but with low molecular diversity) resulting from mulching of Opuntia cladodes. Probably introducing a greater molecular diversity by mulching with more than one organic source, may increase the metabolic demand, and thus potentially limit soil C loss by respiration ${ }^{49}$. However, before considering Firmicutes as markers of low quality soils, we should also consider the role of Bacillus as a plant growth promoter and $\mathrm{P}$ solubilizing agent, especially valuable in arid soils ${ }^{50}$. Only two genera were positively correlated with CEC, namely Rubrobacter (Actinobacteria) and Candidatus Entotheonella (Deltaproteobacteria) both detected in italian soils and both also positively related to soil $\mathrm{pH}$.

Table 3. Spearman correlation coefficients between the relative abundance of the most abundant genera and main soil parameters: cation exchange capacity (CEC), pH, total nitrogen (TN) and soil organic carbon (SOC) obtained in the sampled soils. Only significant correlations ( $p$-value $<0.05$ ) are shown. Blue and red colors indicate negative and positive relationships, respectively, with color intensity representing the degree of significance $(p<0.05>0.01,<0.01>0.001$ and $<0.001)$. 


\begin{tabular}{|c|c|c|c|c|c|}
\hline Phylum & Genera & CEC & pH & $\mathbf{T N}$ & SOC \\
\hline \multirow{2}{*}{ Acidobacteria } & Candidatus Koribacter & -0.52 & -0.69 & & \\
\hline & Candidatus Solibacter & -0.46 & -0.78 & & \\
\hline \multirow{5}{*}{ Actinobacteria } & Arthrobacter & & 0.66 & & \\
\hline & Mycobacterium & & -0.48 & & \\
\hline & Pseudonocardia & & 0.68 & & \\
\hline & Rubrobacter & 0.48 & 0.84 & & \\
\hline & Streptomyces & -0.55 & & -0.6 & -0.6 \\
\hline \multirow{5}{*}{ Alphaproteobacteria } & Balneimonas & & 0.86 & & \\
\hline & Bradyrhizobium & & -0.66 & & \\
\hline & Kaistobacter & & & & -0.54 \\
\hline & Rhodoplanes & & -0.66 & & 0.48 \\
\hline & Skermanella & & 0.88 & & \\
\hline \multirow{3}{*}{ Bacteroidetes } & Adhaeribacter & & 0.83 & & \\
\hline & Flavisolibacter & & 0.48 & & \\
\hline & Rhodocytophaga & 0.57 & 0.7 & & \\
\hline Betaproteobacteria & Burkholderia & & -0.82 & & \\
\hline \multirow{2}{*}{ Deltaproteobacteria } & Candidatus Entotheonella & 0.63 & 0.84 & & \\
\hline & Geobacter & & & & \\
\hline \multirow{6}{*}{ Firmicutes } & Alicyclobacillus & & & & \\
\hline & Ammoniphilus & -0.77 & & & -0.63 \\
\hline & Bacillus & -0.57 & & & -0.71 \\
\hline & Planifilum & & & -0.47 & \\
\hline & Solibacillus & & & & -0.55 \\
\hline & Sporosarcina & -0.51 & & & -0.52 \\
\hline \multirow{2}{*}{ Gammaproteobacteria } & Pseudomonas & & 0.62 & & \\
\hline & Steroidobacter & & 0.84 & & \\
\hline Nitrospirae & Nitrospira & & 0.73 & & \\
\hline \multirow{2}{*}{ Verrucomicrobia } & Candidatus Xiphinematobacter & & & & \\
\hline & $D A 101$ & -0.57 & -0.51 & & \\
\hline
\end{tabular}

\section{The core microbiota of semiarid Mediterranean soils}

The soil core microbiota was identified as phyla and genera that exist in all the samples with at least $1 \%$ of the relative abundance within each sample, respectively ${ }^{41}$. At the phylum level the core microbiota of semi-arid soils under desertification risk of southern Europe (considering exclusively those from sites with an $\mathrm{Al}<0.65)$, was composed of four phyla Proteobacteria, Actinobacteria, Acidobacteria and Firmicutes (Fig. 3). At genus level we were unable to define a common core microbiota, due to high variability of 
edaphic parameters. At regional level Bacillus, Rubrobacter and Balneimonas were the (identified) core microbiota in Sicilian soils (Al 0.29-0.49) (Fig. 4). While Bacillus is an ubiquitarious genus, Rubrobacter, belonging to a deep evolutionary line of descent in the class of Actinobacteria is known for its multiextremophilic growth conditions. Although it was not possible to classify it at species level, this genus is highly represented in extremely hot, arid and/or acidic ecosystems or habitats with severe

radiation/desiccation conditions, such as deserts, volcanic areas and other arid regions ${ }^{30} 51$. Its presence was recently associated to high polyphenol oxidase activities and accumulation of polyhydroxyalkanoates that could function as protection against stress factors ${ }^{38}$. Balneimonas (Bradyrhizobiaceae) is also associated to arid soils where it produces extracellular material that contributes to the formation of soil crusts ${ }^{52} ; 53$.

The core microbiota of Alentejo soils (Al 0.35-0.38) beyond Bacillus, included: DA101, Candidatus Solibacter (Acidobacteria), and Rhodoplanes (Fig.4). The phylotype DA101 within the, as yet, underdescribed soil phylum Verrucomicrobia, has been reported in a wide range of soils and ecosystem types throughout the world. In this work it resulted negatively correlated to CEC, coherently with its previous identification as low fertility specialist ${ }^{54}$. Similarly, Candidatus Solibacter belonging to slow growing oligotrophs adapted to resource limitations ${ }^{35}$, is strongly negatively correlated to soil $\mathrm{pH}$ and CEC (Table 2 and 3). This genus reported as dominant in arid soils ${ }^{53}$, was abundant also in coniferous forest soils of our study sites. Rhodoplanes is a phototrophic ${ }^{53}$, nitrogen fixing genus ${ }^{55}$.

These traits, together with the positive correlation with SOC, suggest a pivotal role of this genus in low nutrient Mediterranean semi-arid soils where it may be involved in carbon and nitrogen fixation. Rhodoplanes was also the only genus represented above $0.8 \%$ in all investigated soils. Thus, Rhodoplanes could be considered "the rare endemic genus" within arid Mediterranean soils where drought reduces the dominance of common bacterial species and allow rare soil bacteria suited for resource limited environments to proliferate 5657 .

\section{Conclusions}

This work provides the first large scale overview of the soil bacterial diversity across European Soils threatened by desertification in the Mediterranean regions.

Overall the results obtained so far suggest that the impact of desertification and soil degradation on the soil microbiota, could be fundamentally different from previous knowledge for well-studied plants and animals ${ }^{58}$. According to our results increasing aridity (that means lowering the $\mathrm{Al}$ ) due to rain reduction and increased temperatures, will lead to a reduction in both SOC and microbial biomass with consequential effects on soil ecosystem services ${ }^{9}$. Conversely bacterial diversity, that did not correlate to the soil organic carbon content, nor to cation exchange capacity, will be affected in terms of community structure and composition but not necessarily as alfa diversity. It appears that microbial biomass rather than diversity is the soil biological index most appropriate to indicate the negative effect of desertification 
on soil biology. The main edaphic driver of bacterial diversity, in the analyzed sites, was $\mathrm{pH}$, an intrinsic soil parameter that was confirmed positively related to biodiversity. Beyond soil $\mathrm{pH}, \mathrm{SOC}$ and $\mathrm{CEC}$ exerted the strongest influence on the soil bacterial community structure, which was also influenced by biogeography, as bacterial community similarity decreased significantly with increasing geographic distance.

Among soil cover, croplands displayed the lowest microbial biomass while shrublands hosted the lowest diversity. In contrast, coniferous forest soil displayed highest beta-diversity irrespectively of aridity index levels and biogeography than generally reported, and this could contribute to higher resilience to drought of these ecosystems in respect to other soil covers.

Overall it appears difficult to describe a core microbiota of Mediterranean semi-arid soils because microbial responses to increasing warming and drought across soils and regions can be different and related to other edaphic and environmental parameters.

Future modifications induced by global change factors in the microbial community structure may have different impact on soil functionality by affecting the abundance of relevant phyla such as Actinobacteria, Bacteroidetes and Proteobacteria and by selecting different resistant and resilient bacterial taxa, such as the rare multi-stress resistant genera that make up the (identified) core microbiota of semi-arid European soils. The genome of the core members Rhodoplanes, Rubrobacter, Balneimonas, and phyloptype DA101 endowed with highly specific traits to cope with low nutrient, dry, harsh conditions, could reveal ecofunctional strategies that can be exploited for arid land reclamation. Even more could be revealed by metagenomics and metaproteomics approaches that will shed light also on the taxa that were not identified by $16 \mathrm{~S}$ metagenomics.

The results of this investigation will be useful as baseline for the necessary monitoring of European soils facing the intensification of climate change and for the evaluation of the efficacy of adaptation measures to contrast desertification.

\section{Materials And Methods}

\section{Soil sampling from the study sites}

Top soil was sampled $(0-10 \mathrm{~cm})$ from 30 sites, situated in 10 municipalities, either private farms or public areas, in southern Portugal (Alentejo, PT), Central Spain (Extremadura, SP) and Southern Italy (Sicily, IT) during the Spring $2018^{10}$ (Table S1). The sites included different land covers/uses, grouped in 4 main dominant cover ${ }^{10}$ : trees, pastures dominated by grass cover, shrublands and croplands, In each plot, from 1 to 3 sub-samples were collected in sterile plastic bags and kept refrigerated. Once in the laboratory (after 1-4 days from sampling) sub-samples were pooled, to obtain 86 samples that were immediately subjected to DNA extraction. Soil subsamples were oven dried at $65^{\circ} \mathrm{C}$ up to constant weight.

\section{Metagenomic DNA Extraction from soil samples and estimation of microbial biomass}

Page $13 / 25$ 
The total soil DNA was extracted from aliquots of $0.5 \mathrm{~g}$ of fresh soil sample with the FastDNA ${ }^{\text {TM }}$ SPIN Kit for Soil (MP Biomedicals, Germany) using the FastPrep instrument, according to the manufacturer's instructions, with exception in the last step, where the nucleic acids were eluted as described in Novara et al., $2020^{30}$. Metagenomic DNAs were checked for quality and concentration and stored at $-20^{\circ} \mathrm{C}$ until further processing.

\section{Automated Ribosomal Intergenic Spacer Analysis (ARISA)}

The soil bacterial communities were investigated using automated ribosomal intergenic spacer (ITS) analysis. Soil DNA extracted as described above was used as a template in PCR amplifications for automated ribosomal intergenic spacer analysis (ARISA) using universal primers ITSF/ITSReub targeting the 16S-23S rRNA ITS ${ }^{59}$ ITSReub were labelled with the phosphoramidite dye HEX (6-carboxy-1,4dichloro-2,4,5,7-tetra-chlorofluorescein). ARISA-PCR was performed following the method of Cardinale et al., 2004, with modifications. Reaction mixtures contained OneTaq Standard Reaction Buffer 1 X (NEB), a $200 \mu \mathrm{M}$ concentration of dNTPs mixture (Invitrogen), a $250 \mu \mathrm{M}$ concentration of each primer, $1 \mathrm{U}$ of OneTaq DNA Polymerase (NEB), and approximately $10 \mathrm{ng}$ of template metagenomic DNA in a final volume of $50 \mu \mathrm{l}$. Reaction mixtures were held at $94^{\circ} \mathrm{C}$ for $30 \mathrm{~s}$, followed by 30 cycles of amplification at $94^{\circ} \mathrm{C}$ for $30 \mathrm{~s}, 55^{\circ} \mathrm{C}$ for $1 \mathrm{~min}$, and $68^{\circ} \mathrm{C}$ for $1 \mathrm{~min}$ and a final extension of $68^{\circ} \mathrm{C}$ for $5 \mathrm{~min}$. The best concentration of labelled PCR product was tested and estimated by comparing it to known quantities of PCR products and by run a dilution series on Bioanalyzer ABI PRISM® 310 Genetic Analyzer. After determining the optimal dilution ratio, the same dilutions for all ITS products was utilized. Based on these estimates, a standardized amount (between 0.5 to $4 \mu \mathrm{l}$ ) of PCR product was used for ARISA. Amplified ITS fragments were separated on a capillary electrophoresis Bioanalyzer ABI PRISM® 310 Genetic Analyzer (Applied Biosystems, Foster City, CA, USA) according to the manufacturer's instructions. Run conditions were performed as described elsewhere ${ }^{60}$. The output of ARISA was analyzed by the Peak Scanner software vs1.0 and the biodiversity indices were calculated using the PAST 3.0 software (http://folk.uio.no/ohammer/past). Fragments in the range between 50 and 1000 bp were analysed and exported into spreadsheets for subsequent analysis, the fluorescence threshold was set at 50 relative fluorescence units (RFU). A bin size of 5 bp was employed to minimise the inaccuracies in the ARISA profiles.

\section{Illumina High-Throughput Analysis of 16S rRNA gene and data processing}

The V3-V4 hypervariable region of prokaryotic 16S rRNA gene, for the simultaneous detection of bacteria and archaea, was sequenced using Miseq Illumina with 300bp paired-end reads. PCR amplification was performed in a $25 \mu$ reaction volume containing $10 \mathrm{ng}$ of metagenomic DNA, MightyAmp for Real Time (SYBR Plus) (Takara, Japan), $0.25 \mathrm{mM}$ of Pro341F/Pro805R primers ${ }^{61}$. The PCR procedure was as follows: an initial cycle of $2 \mathrm{~min}$ at $98^{\circ} \mathrm{C}$, followed by 35 cycles of annealing beginning at $65^{\circ} \mathrm{C}$ and ending at $55^{\circ} \mathrm{C}$ for $15 \mathrm{sec}$ (the temperature was lowered $1^{\circ} \mathrm{C}$ every cycle), and extension at $68^{\circ} \mathrm{C}$ for 30 sec. Sequences obtained from Illumina Sequencing were processed using the QIIME2 software package version $2018.4^{62}$. To find the reads containing adapters and to remove the adapters Cutadapt software 
version v2.9 was used. Quality check, trimming, error rate estimation, dereplication and read merging were done using the DADA2 a R packages wrapped in QIIME2 ${ }^{63}$. To remove chimeras from the lllumina sequenced FASTQ files the "consensus" method implemented in DADA2 was used. For taxa assignment the QIIME2 q2-feature-classifier plugin that was trained on the Greengenes database version 13-8 with a 99\% Operational Taxonomic Units (OTUs) full-length sequences. QIIME2 taxa barplot command and ggplot2 were used for visualization of the taxonomic composition of the samples.

The 16S rDNA amplicon rRNA gene sequences were deposited into the NCBI short reads archive (SRA) database under BioProject number .....

\section{Statistical analysis}

To test the effects of soil physical-chemical properties (SOC, pH, TN and CEC) on SMB, richness and diversity we used multiple linear regression. Soil properties data were obtained from ${ }^{10}$ and refereed to the same soil samples, which were split at the moment of sampling, after thorough mixing, among different research groups of the project. We first performed ordinary least square regression and checked the residuals of the models for normality with a Shapiro-Wilk test. The regression residuals for Shannon diversity index were not normal $(p<0.001)$ and once the data could not be normalized with common transformations, we decided to exclude 4 outliers with very low values ranging from 1.40 to 1.75 (one sample in SP3-2, two samples in SP3-3 and one in PT 2-4), obtaining then a normal residual distribution $(p=0.061)$. To test the differences across the three countries and five land uses we used ANOVA with Tukey HSD post-hoc test for richness and Kruskall-Wallis with Dunn post-hoc test for SMB and Shannon diversity index. We also tested for differences in richness ( $t$ test), SMB and diversity (Wilcoxon test) across two groups of sites, those containing SOC < than $20 \mathrm{~g} \mathrm{~kg}^{-1}$ soil $(n=31)$ and those with higher values $(n=38)$, following the threshold reported in Grilli et al. $(2021)^{10}$ as critical for desertification.

Multivariate analysis of community structure and (beta)diversity was performed on the ARISA-based dataset and soil variables using Canonical Analysis of Principal Coordinates CAP with PRIMER v7 64 with the PERMANOVA+ add-on package ${ }^{64}$.

To check for differences in the relative abundancies of bacterial phyla between countries and soil use, we used ANOVA and post hoc Tukey HSD tests for the normally distributed data (after Shapiro-Wilk test on the residuals) and Kruskal-Wallis with post hoc Dunn test for the non-normally distributed phyla (Bacteriodetes, Chloroflexi e Firmicutes). We also compared the relative abundances between sites with more $(n=14)$ and less $(n=6)$ than $20 \mathrm{~g} \mathrm{~kg}^{-1}$ of SOC.

We used multiple regressions to test the relationship of soil physical-chemical variables ( $\mathrm{pH}, \mathrm{SOC}, \mathrm{TN}$, CEC) with the relative abundance of the most represented phyla. For Bacteroidetes, Chloroflexi and Firmicutes we fitted generalized linear models with negative binomial distribution and log link functions to control for the overdispersion found in the Poisson models. For the other taxa we used ordinary least square regressions. After fitting the models, we checked for multicollinearity among the predictors using 
the Variance Inflation Factor, with all values below 4.3. Statistical analysis was performed with R v 3.6.1 (R core Team 2019).

To check for the relationship between aridity index (the ratio of annual precipitation to annual potential evapotranspiration) and SMB, richness and Shannon diversity, the sites were classified in two groups: semi-arid (aridity index $>0.2$ and $<0.5$ ) or not-arid $\left(>0.65\right.$ ), accordingly to the Aridity Index rank ${ }^{5}$. Differences across the two groups were assessed with Wilcoxon rank test for SMB and Shannon index and $t$ tests for richness. A lower Al value indicate a higher degree of aridity.

\section{Declarations}

\section{Acknowledgements}

The present study was supported by the EU project LIFE16 CCA/IT/000011. We thank Marco Milazzo for CAP analysis and Marco Tolone for support in bioinformatics analysis.

\section{Conflicts of interest/Competing interests}

V.C. declares that she has no conflict of interest/Competing interests. R.S.B. declares that he has no conflict of interest/ Competing interests. T.L.M. declares that he has no conflict of interest/ Competing interests. R.A. declares that she has no conflict of interest/ Competing interests. S.C. declares that she has no conflict of interest / Competing interests. E.G. declares that she has no conflict of interest / Competing interests. P.Q. declares that she has no conflict of interest / Competing interests

\section{Authors' contributions}

V.C. designed and performed the molecular analysis and bioinformatics and wrote the first draft; R.S.B. collected soil samples, carried out statistics and wrote the first draft of the manuscript; R.A. carried out laboratory work and elaborated the data, T.L.M., S.C. and E.G. designed the sampling campaign, collected soil samples, disclosed physical chemical data. P.Q. performed the study conception and design, data collection and analysis and wrote the first draft of the manuscript. All authors contributed and commented on previous versions of the manuscript, read and approved the final manuscript.

\section{References}

1. Delgado-Baquerizo, M. et al. It is elemental: soil nutrient stoichiometry drives bacterial diversity. Environ. Microbiol. 19, 1176-1188 (2017).

2. Yang, T., Siddique, K. H. M. \& Liu, K. Cropping systems in agriculture and their impact on soil health-A review. Glob. Ecol. Conserv. 23, e01118 (2020).

3. Stolte, J. et al. Soil threats in Europe. EUR 27607. JRC Scientific and Technical Reports (2016).

4. Amend, A. From Dandruff to Deep-Sea Vents: Malassezia-like Fungi Are Ecologically Hyper-diverse. PLoS Pathog. 10, e1004277 (2014). 
5. Middleton, N. \& Thomas, D. S. G. World Atlas of Desertification. First. (1992).

6. Wiesmeier M. Environmental Indicators of Dryland. Environ. Indic. 239-250 (2015).

7. Tecon, R. \& Or, D. Biophysical processes supporting the diversity of microbial life in soil. FEMS Microbiol. Rev. 41, 599-623 (2017).

8. Maestre, F. T. et al. Increasing aridity reduces soil microbial diversity and abundance in global drylands. Proc. Natl. Acad. Sci. U. S. A. 112, 15684-15689 (2015).

9. Bastida, F. et al. Soil microbial diversity-biomass relationships are driven by soil carbon content across global biomes. ISME J. 15, 2081-2091 (2021).

10. Grilli, E. et al. Critical range of soil organic carbon in southern Europe lands under desertification risk. J. Environ. Manage. 287, (2021).

11. Terhonen, E., Blumenstein, K. \& Kovalchuk, A. Forest Tree Microbiomes and Associated Fungal Endophytes: Functional Roles and Impact on Forest Health. 1-32 (2019). doi:10.3390/f10010042

12. Su, Y. et al. Linking soil microbial community dynamics to straw-carbon distribution in soil organic carbon. Sci. Rep. 10, 1-12 (2020).

13. Domeignoz-Horta, L. A. et al. Microbial diversity drives carbon use efficiency in a model soil. Nat. Commun. 11, 1-10 (2020).

14. Timmis, K. et al. The urgent need for microbiology literacy in society. Environ. Microbiol. 21, 15131528 (2019).

15. Hagerty, S. B. et al. Accelerated microbial turnover but constant growth efficiency with warming in soil. Nat. Clim. Chang. 4, 903-906 (2014).

16. Saccá M.L., Barra Caracciolo A., Di Lenola M., G. P. Ecosystem Services Provided By Soil Microorganisms. In: Lukac M., Grenni P., Gamboni M. (eds) Soil Biological Communities and Ecosystem Resilience. Sustain. Plant Crop Prot. Springer, (2017).

17. Bünemann, E. K. et al. Soil quality - A critical review. Soil Biology and Biochemistry (2018). doi:10.1016/j.soilbio.2018.01.030

18. Steidinger, B. S. et al. Climatic controls of decomposition drive the global biogeography of forest-tree symbioses. Nature 569, 404-408 (2019).

19. Dubey, A. et al. Soil microbiome: a key player for conservation of soil health under changing climate. Biodivers. Conserv. 28, 2405-2429 (2019).

20. Hagan, J. G., Vanschoenwinkel, B. \& Gamfeldt, L. We should not necessarily expect positive relationships between biodiversity and ecosystem functioning in observational field data. Ecol. Lett. 24, 2537-2548 (2021).

21. Hendershot, J. N., Read, Q. D., Henning, J. A., Sanders, N. J. \& Classen, A. T. Consistently inconsistent drivers of microbial diversity and abundance at macroecological scales. Ecology $98,1757-1763$ (2017).

22. Plassart, P. et al. Soil parameters, land use, and geographical distance drive soil bacterial communities along a European transect. Sci. Rep. 9, 1-17 (2019). 
23. ECA. Combating desertification in the EU: a growing thread in need for more action. Eur. Court Audit. 33, (2018).

24. Dequiedt, S. et al. Biogeographical patterns of soil molecular microbial biomass as influenced by soil characteristics and management. Glob. Ecol. Biogeogr. 20, 641-652 (2011).

25. Fornasier, F., Ascher, J., Ceccherini, M. T., Tomat, E. \& Pietramellara, G. A simplified rapid, low-cost and versatile DNA-based assessment of soil microbial biomass. Ecol. Indic. 45, 75-82 (2014).

26. Ren, C. et al. Responses of soil total microbial biomass and community compositions to rainfall reductions. Soil Biol. Biochem. 116, 4-10 (2018).

27. Leff, J. W. et al. Consistent responses of soil microbial communities to elevated nutrient inputs in grasslands across the globe. Proc. Natl. Acad. Sci. U. S. A. 112, 10967-10972 (2015).

28. Gleixner, G. Soil organic matter dynamics: A biological perspective derived from the use of compound-specific isotopes studies. Ecol. Res. 28, 683-695 (2013).

29. Cotrufo, M. F., Wallenstein, M. D., Boot, C. M., Denef, K. \& Paul, E. The Microbial Efficiency-Matrix Stabilization (MEMS) framework integrates plant litter decomposition with soil organic matter stabilization: Do labile plant inputs form stable soil organic matter? Glob. Chang. Biol. 19, 988-995 (2013).

30. Novara, A. et al. Cover crop impact on soil organic carbon, nitrogen dynamics and microbial diversity in a mediterranean semiarid vineyard. Sustain. 12, (2020).

31. Xie, J. et al. Rapid accumulation of carbon on severely eroded red soils through afforestation in subtropical China. For. Ecol. Manage. 300, 53-59 (2013).

32. Goberna, M., Sánchez, J., Pascual, J. A. \& García, C. Pinus halepensis Mill. plantations did not restore organic carbon, microbial biomass and activity levels in a semi-arid Mediterranean soil. Appl. soil Ecol. 36, 107-115 (2007).

33. Grilli, E. et al. Soil Quality Characterization of Mediterranean Areas under Desertification Risk for the Implementation of Management Schemes Aimed at Land Degradation Neutrality. Proceedings 30, 54 (2020).

34. Rutigliano, F. A., D’ascoli, R. \& De Santo, A. V. Soil microbial metabolism and nutrient status in a Mediterranean area as affected by plant cover. Soil Biol. Biochem. 36, 1719-1729 (2004).

35. Lladó, S., López-Mondéjar, R. \& Baldrian, P. Forest Soil Bacteria: Diversity, Involvement in Ecosystem Processes, and Response to Global Change. Microbiol. Mol. Biol. Rev. 81, 1-27 (2017).

36. Ullah, M. R., Carrillo, Y. \& Dijkstra, F. A. Drought-induced and seasonal variation in carbon use efficiency is associated with fungi: bacteria ratio and enzyme production in a grassland ecosystem. Soil Biol. Biochem. 155, 108159 (2021).

37. Bastida, F. et al. The active microbial diversity drives ecosystem multifunctionality and is physiologically related to carbon availability in Mediterranean semi-arid soils. Mol. Ecol. 25, 46604673 (2016). 
38. Moreno; josé Luis, Torres, I. F., García, C., López-Mondéjar, R. \& Bastida, F. Land use shapes the resistance of the soil microbial community and the $\mathrm{C}$ cycling response to drought in a semi-arid area. Sci. Total Environ. 648, 1018-1030 (2019).

39. Cotrufo, M. F., Ranalli, M. G., Haddix, M. L., Six, J. \& Lugato, E. Soil carbon storage informed by particulate and mineral-associated organic matter. Nat. Geosci. 12, 989-994 (2019).

40. Delgado-Baquerizo, M. et al. Bacteria Found in Soil. Science (80-. ). 325, 320-325 (2018).

41. Qi, D., Wieneke, X., Tao, J., Zhou, X. \& Desilva, U. Soil pH is the primary factor correlating with soil microbiome in karst rocky desertification regions in the Wushan County, Chongqing, China. Front. Microbiol. 9, 1-12 (2018).

42. Terrat, S. et al. Improving soil bacterial taxa-area relationships assessment using DNA metabarcoding. Heredity (Edinb). 114, 468-475 (2015).

43. Hazelton, P. \& Murphy, B. Interpreting Soil Test Results: What do all the Mean? (CSIRO Publishing. Collingwood Victoria - Australia. http://www.publish.CSIRO., 2007).

44. Fierer, N., Bradford, M. A. \& Jackson, R. B. Toward an ecological classification of soil bacteria. Ecology 88, 1354-1364 (2007).

45. Whitman, T. et al. Dynamics of microbial community composition and soil organic carbon mineralization in soil following addition of pyrogenic and fresh organic matter. ISME J. 10, $2918-$ 2930 (2016).

46. Singh, B. K., Bardgett, R. D., Smith, P. \& Reay, D. S. Microorganisms and climate change: Terrestrial feedbacks and mitigation options. Nat. Rev. Microbiol. 8, 779-790 (2010).

47. Ho, A., Di Lonardo, D. P. \& Bodelier, P. L. E. Revisiting life strategy concepts in environmental microbial ecology. FEMS Microbiol. Ecol. 93, (2017).

48. Ranney, M. A. \& Clark, D. Climate Change Conceptual Change: Scientific Information Can Transform Attitudes. Top. Cogn. Sci. 8, 49-75 (2016).

49. Lehmann, J. et al. Persistence of soil organic carbon caused by functional complexity. Nat. Geosci. doi:10.1038/s41561-020-0612-3

50. Ayangbenro, A. S. \& Babalola, O. O. Reclamation of arid and semi-arid soils: The role of plant growthpromoting archaea and bacteria. Current Plant Biology 25, (2021).

51. Kourilová, X. et al. The First Insight into Polyhydroxyalkanoates Accumulation in Multi-Extremophilic Rubrobacter xylanophilus and Rubrobacter spartanus. Microorganisms 9, 909 (2021).

52. Matthews, A., Pierce, S., Hipperson, H. \& Raymond, B. Rhizobacterial community assembly patterns vary between crop species. Front. Microbiol. 10, 581 (2019).

53. Khan, M. A. \& Khan, S. T. Microbial communities and their predictive functional profiles in the arid soil of Saudi Arabia. Soil 6, 513-521 (2020).

54. Fierer, N. et al. Prairie Soils in the United States. Science (80-. ). 342, 621-625 (2013).

55. Buckley, D. H., Huangyutitham, V., Hsu, S. F. \& Nelson, T. A. Stable isotope probing with 15N2 reveals novel noncultivated diazotrophs in soil. Appl. Environ. Microbiol. 73, 3196-3204 (2007). 
56. Vohník, M. et al. The cultivable endophytic community of Norway spruce ectomycorrhizas from microhabitats lacking ericaceous hosts is dominated by ericoid mycorrhizal Meliniomyces variabilis. Fungal Ecol. 6, 281-292 (2013).

57. Bickel, S. \& Or, D. The chosen few-variations in common and rare soil bacteria across biomes. ISME J. 1-11 (2021).

58. Zhou, Z., Wang, C. \& Luo, Y. Meta-analysis of the impacts of global change factors on soil microbial diversity and functionality. Nat. Commun. 11, (2020).

59. Cardinale, M. et al. Comparison of different primer sets for use in automated ribosomal intergenic spacer analysis of complex bacterial communities. Appl. Environ. Microbiol. 70, 6147-6156 (2004).

60. La Marca, E. C. et al. Temporal dynamic of biofilms enhances the settlement of the centralMediterranean reef-builder Dendropoma cristatum (Biondi, 1859). Mar. Environ. Res. 172, 105484 (2021).

61. Takahashi, S., Tomita, J., Nishioka, K., Hisada, T. \& Nishijima, M. Development of a prokaryotic universal primer for simultaneous analysis of Bacteria and Archaea using next-generation sequencing. PLoS One 9, (2014).

62. Caporaso JG, Kuczynski J, Stombaugh J, Bittinger K, Bushman FD, Costello EK, Fierer N, Peña AG, Goodrich JK, Gordon JI, Huttley GA, Kelley ST, Knights D, Koenig JE, Ley RE, Lozupone CA, McDonald D, Muegge BD, Pirrung M, Reeder J, Sevinsky JR, Turnbaugh PJ, K. R. QIIME allows analysis of highthroughput community sequencing data. Nat Methods 7, 335-336 (2010).

63. Callahan, B. J., McMurdie, P. J., Rosen, M. J., Han, A. W., Johnson, A. J. A., \& Holmes, S. P. DADA2: high-resolution sample inference from Illumina amplicon data. Nat. Methods 13, 581-583 (2016).

64. Clarke, K. R. \& Gorley, R. N. Getting started with PRIMER v7. Prim. Plymouth, Plymouth Mar. Lab. 20, (2015).

\section{Figures}


官 Broad-leaved agroforestry 追 Conifer Forest 追 Cropland 官 Pasture-grassland 追 Shrubland
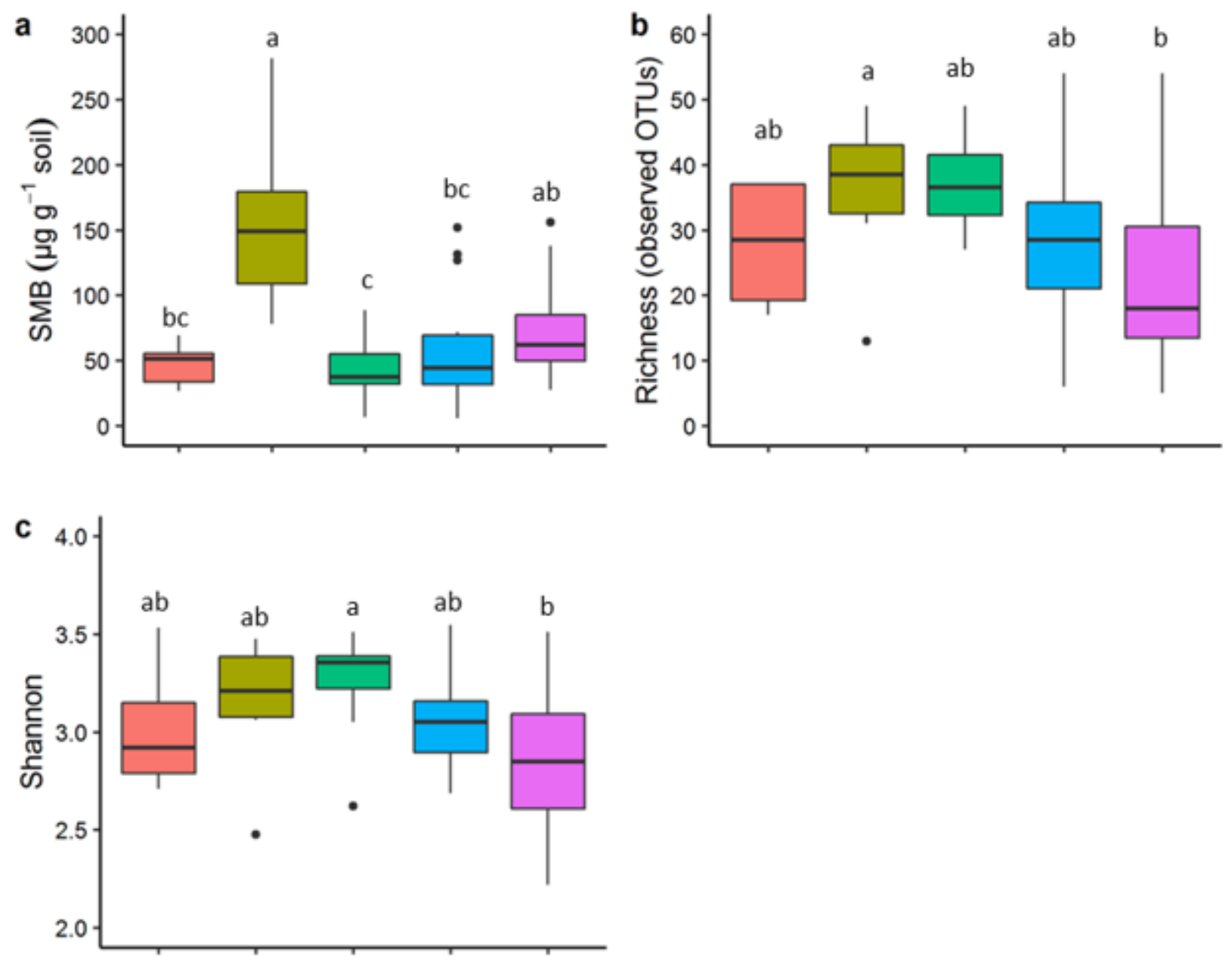

Figure 1

Boxplots (median. interquartile and range) of a) $\operatorname{SMB}(n=86), b)$ richness $(n=69)$ and c) Shannon index $(n=65)$ across the five land uses in the farms of Sicily (Italy), Extremadura (Spain) and Alentejo (Portugal). SMB was estimated as total soil dsDNA, diversity indices were based on ARISA. 


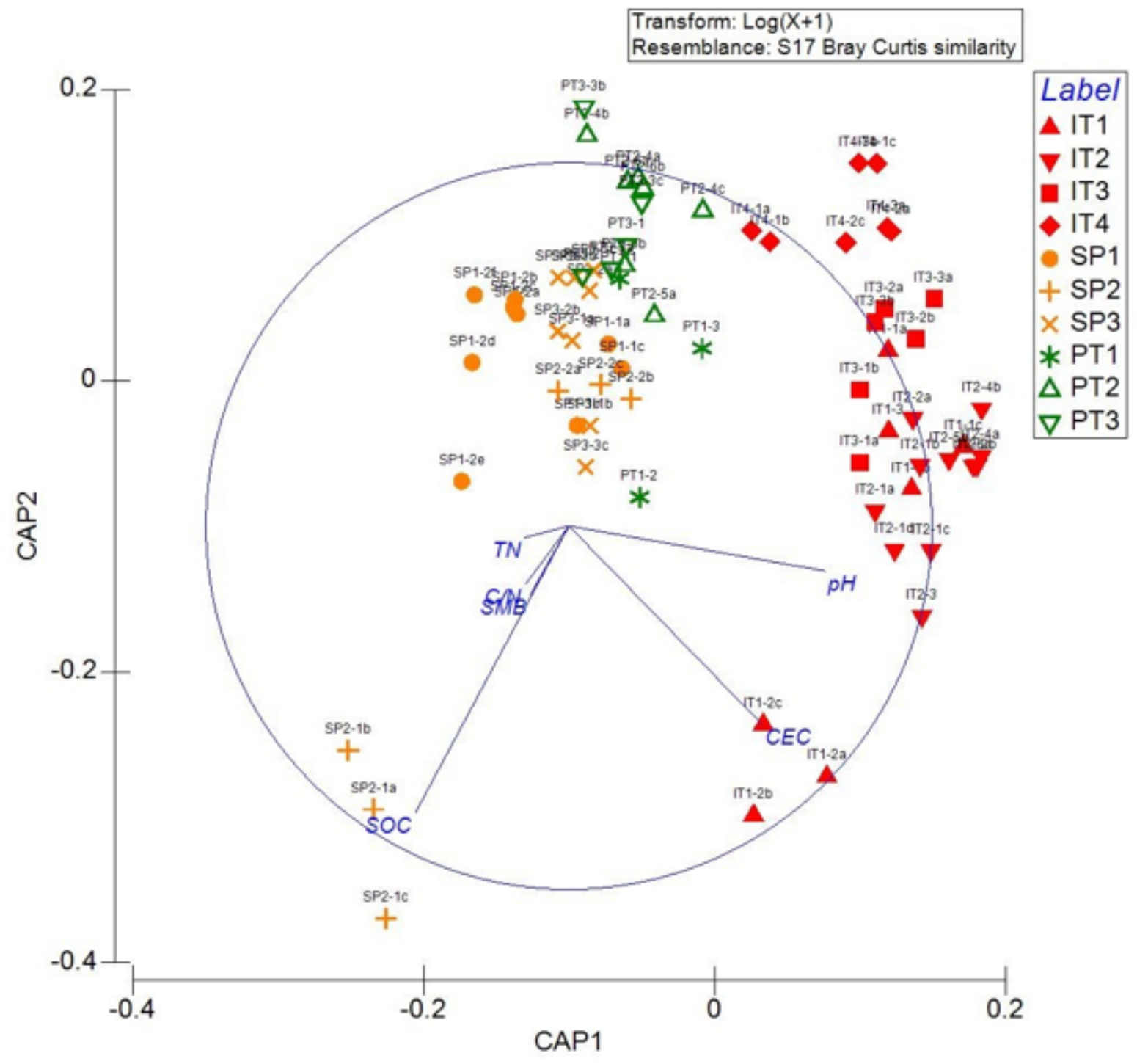

Figure 2

Canonical Analysis of Principal Coordinates (CAP) calculated on the ARISA profiles of 86 soil samples of the ten farms located in Sicily (Italy), Extremadura (Spain) and Alentejo (Portugal) and the relative main chemical physical soil variables. Labels of bacterial communities are described in Table S1. 


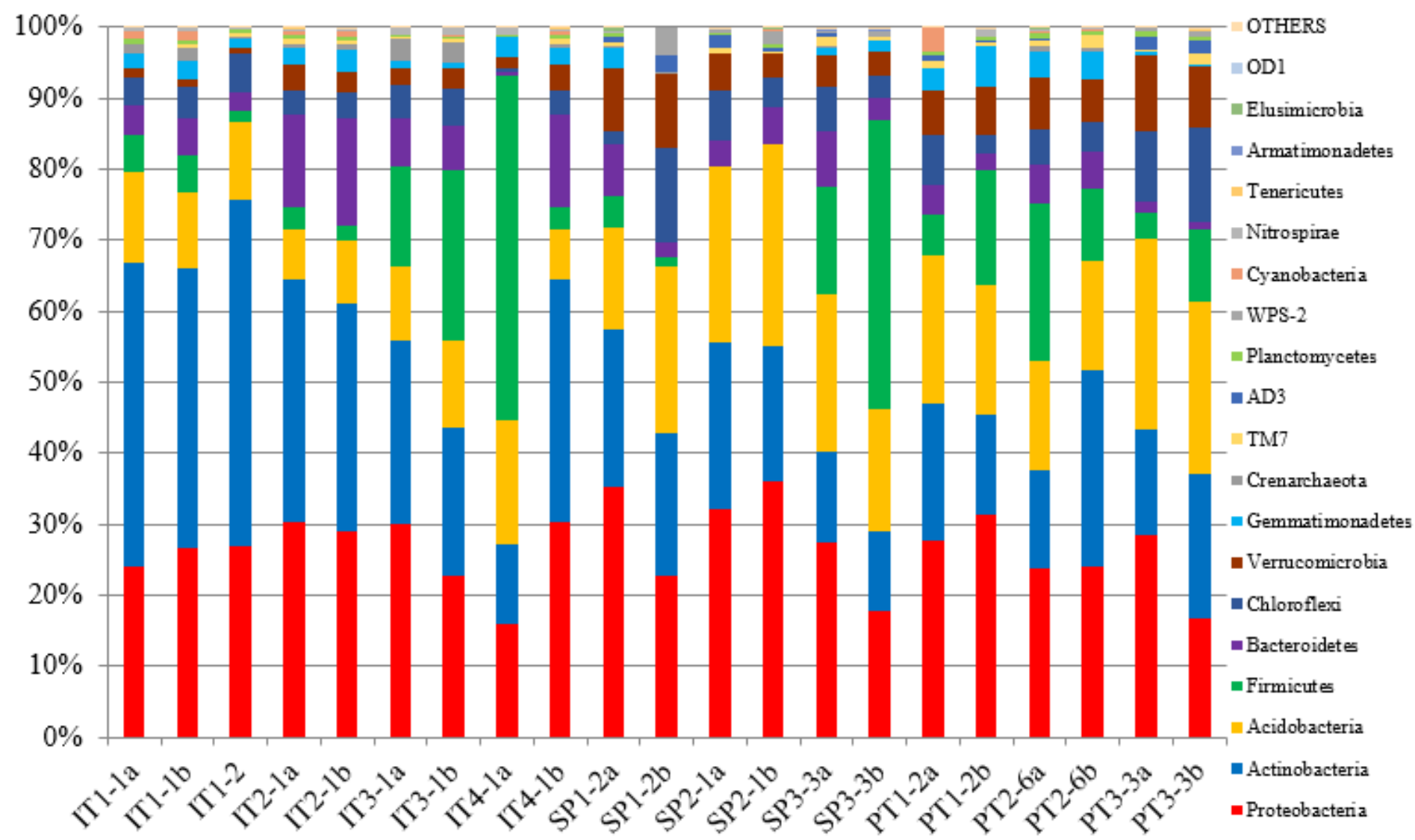

Figure 3

Relative abundance of soil bacterial and archaeal phyla in the semi-arid soils of Southern Europe. Others includes phyla $<0.1 \%$. 


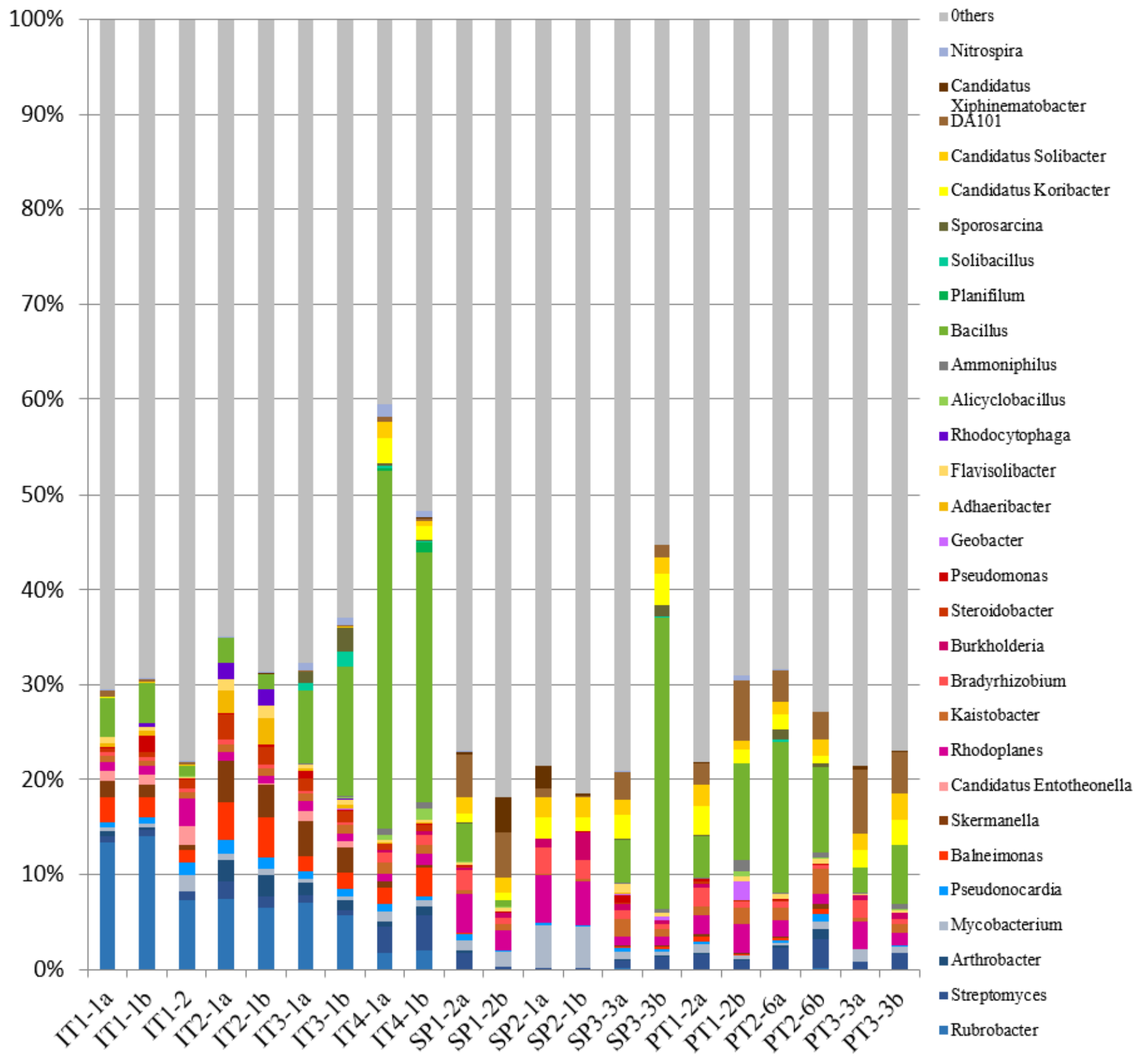

Figure 4

Relative abundance of bacterial genera in the soil communities of desertified ecosystems in Souther Europe. Genera whose abundance is above 1\% in at least one sample are shown. Others includes 292 genera with abundances below $1 \%$ in all sites.

\section{Supplementary Files}


This is a list of supplementary files associated with this preprint. Click to download.

- CataniaetalSupplementaryMaterial.pdf 